\title{
On Reproducing Kernel and Applications
}

\author{
José Claudinei Ferreira and Estela Costa Ferreira \\ Institute of Exact Sciences, Federal University of Alfenas, Alfenas, Brazil \\ Email: jose.ferreira@unifal-mg.edu.br
}

\begin{abstract}
Positive definite or reproducing kernel are common topics in recent branches of mathematics. In this paper we brief review some facts about this subject and prove some technical results related to convergence, representations by using integral operators, embedding properties, denseness and strict positive definiteness. As an application point of view, we close the paper choosing a special basis to approximate solutions to Volterra integral equations.
\end{abstract}

Keywords: Positive definite kernel, reproducing kernel, approximation, denseness.

\section{Introduction}

Let $X$ be a non-empty set and $K: X \times X \rightarrow \mathbb{C}$ a positive definite kernel on $X$, that is, a function satisfying the inequality

$$
\overline{\left[c_{1} \ldots c_{n}\right]}\left[\begin{array}{ccc}
K\left(x_{1}, x_{1}\right) & \ldots & K\left(x_{1}, x_{n}\right) \\
\vdots & \ddots & \vdots \\
K\left(x_{n}, x_{1}\right) & \ldots & K\left(x_{n}, x_{n}\right)
\end{array}\right]\left[\begin{array}{c}
c_{1} \\
\vdots \\
c_{n}
\end{array}\right]=\sum_{i, j=1}^{n} \overline{c_{i}} c_{j} K\left(x_{i}, x_{j}\right) \geq 0
$$

whenever $n \geq 1, x_{1}, x_{2}, \ldots, x_{n}$ is in $X$ and $c_{1}, c_{2}, \ldots, c_{n}$ is in $\mathbb{C}$. This means that the quadratic form (1) is nonnegative, in other words, the Hermitian matrix $\left[K\left(x_{i}, x_{j}\right)\right]_{n \times n}$ has only nonnegative eigenvalues. If these eigenvalues are always positive, then $K$ is a strict positive definite kernel. Particularly, if $\kappa(x)=K(x, x)$, then

$$
0 \leq \kappa(x), \quad|K(x, y)|^{2} \leq \kappa(x) \kappa(y), \quad x, y \in X .
$$

Positive definiteness may be used to guarantee that interpolation problems like

$$
S(f)(x)=\sum_{i=1}^{n} c_{i} K\left(x, x_{i}\right), \quad S(f)\left(x_{i}\right)=y_{i}, \quad i=1,2 \ldots, n,
$$

where $y_{i} \approx f\left(x_{i}\right)$ is given in a table, has a unique solution. It also helps to find this solution by using numerical methods to solve this kind of systems (see [3]). Another thing closely related to this problem is to approximate a given function by another in some nice space like a reproducing kernel Hilbert spaces (please see [4]).

For a fixed $x \in X$, let us write $K^{x}$ to denote the function $y \in X \rightarrow K(y, x) \in \mathbb{C}$. The unique Hilbert space $\mathcal{H}_{K}$ containing the set

$$
\left\{K^{x}: x \in X\right\}
$$

with inner product given by

$$
\left\langle K^{x}, K^{y}\right\rangle_{K}=K(y, x), \quad x, y \in X,
$$

is the reproducing kernel Hilbert space (RKHS) $\mathcal{H}_{K}$ with the reproducing property:

$$
f(x)=\left\langle f, K^{x}\right\rangle_{K}, \quad x \in X, \quad f \in \mathcal{H}_{K} .
$$

Please see references $[2,3,9,11,12,13]$ to more information on this subject.

This paper is organized as follows. In Section 2 we discuss some technical results on positive definite and reproducing kernel (Hilbert spaces). In Section 3 we give the representation of solutions of "linear" equations in reproducing kernel spaces. Section 4 is devoted to closing the paper talking about an appropriate space to apply the previous results to approximate solutions of Volterra integral equations. 


\section{On Reproducing Kernel Properties}

We can talk about RKHS by using another point of view, as we briefly do next. Let $\mathcal{H}$ be a Hilbert space of functions $f: X \rightarrow \mathbb{C}$ for which the evaluation linear functional $\delta_{x}: \rightarrow \mathbb{C}$, given by

$$
\delta_{x}(f)=f(x), \quad f \in \mathcal{H}, \quad x \in X,
$$

is bounded. As we know, this means that

$$
|f(x)|=\left\|\delta_{x}(f)\right\|_{\mathcal{H}} \leq C_{x}\|f\|_{\mathcal{H}}, \quad f \in \mathcal{H}, \quad x \in X,
$$

to some constants $C_{x} \geq 0$.

This and Riesz representation theorem produce the next well known result.

Lemma 2.1. The space $\mathcal{H}$ is a RKHS if, and only if, the all evaluation functional $\delta_{x}, x \in X$, is bounded. Also,

$$
\left\|\delta_{x}\right\|=\left\|K^{x}\right\|_{K}=\sqrt{\left\langle K^{x}, K^{x}\right\rangle_{K}}=\sqrt{K(x, x)}, \quad x \in X,
$$

and the reproducing kernel is unique.

Next results are important in approximation in RKHS (see $[11,13]$ ). We write $C(X)$ to denote the set of all complex continuous functions on $X$.

Lemma 2.2. If $f_{n} \rightarrow f$ in $\mathcal{H}_{K}$. Then $f_{n}(x) \rightarrow f(x)$, uniformly on $Y \subset X$, when $\sup _{x \in Y} \kappa(x)<\infty$.

Proof. We just need to note that, if $x \in X$ then

$$
\left|f_{n}(x)-f(x)\right|=\left|\delta_{x}\left(f_{n}\right)-\delta_{x}(f)\right|=\left|\delta_{x}\left(f_{n}-f\right)\right| \leqslant\left\|\delta_{x}\right\|\left\|f_{n}-f\right\|_{\mathcal{H}} .
$$

Lemma 2.3. If $\mathcal{H}_{K} \subset C(X)$ and $\left\{x_{i}\right\}_{i \in A}$ is a dense set in $X$. Then the linear span of $\left\{K\left(\cdot, x_{i}\right)\right\}_{i \in A}$ is dense in $\mathcal{H}_{K}$. This set is always linearly independent if, and only if, $K$ is strict positive definite.

Proof. Let $f \in \mathcal{H}_{K}$ be a continuous function and orthogonal to the set $\left\{K^{x_{i}}\right\}_{i \in A}$. It follows that

$$
f\left(x_{i}\right)=\left\langle f, K^{x_{i}}\right\rangle_{K}=0
$$

and hence $f=0$. To finish just note that $K$ is strict positive definite if, and only if,

$$
g=\sum_{i=1}^{n} c_{i} K\left(x_{i}, \cdot\right)=0 \Longleftrightarrow\langle g, g\rangle_{K}=\sum_{i, j=1}^{n} c_{i} \overline{c_{j}} K\left(x_{i}, x_{j}\right)=0 \Longleftrightarrow c_{i}=0, i=1,2, \ldots, n .
$$

A way to check if a Hilbert space is a reproducing kernel space is given in the next theorem.

Theorem 2.4. Let $\mathcal{F}(X)$ be an inner product space of functions for which $\delta_{x}$ is bounded, for all $x \in X$. The a Hilbert space containing $\{\mathcal{F}(X)\}$ is a RKHS if, and only if, the convergence in this space implies the pointwise convergence.

Proof. Let $\mathcal{H}$ be a Hilbert space containing $\mathcal{F}(X)$. If $\left\|f_{n}-f\right\|_{\mathcal{H}} \rightarrow 0$ implies that $f_{n}(x) \rightarrow f(x)$ then our hypothesis guarantees that $|f(x)| \leq C_{x}\|f\|_{\mathcal{H}}$ and $\mathcal{H}$ is a RKHS. The other side is trivial.

If we are working with a reproducing kernel Hilbert space $\mathcal{H}_{K}$, then on important property to check is if it has continuous functions only. To analyze this question, we say that the set of functions $\left\{K^{x}: x \in X\right\}$ is locally equicontinuous if, for all $x \in X$, there is an open neighbourhood $U_{x}$ such that $\left\{K^{y}: y \in U_{x}\right\}$ is equicontinuous.

The continuity of $K$ is related to that of the feature map $\eta: X \rightarrow \mathcal{H}_{K}$ given by

$$
\eta(x)=K^{x}, \quad x \in X .
$$

Also, there is an equivalence between the continuity of $K$ and that of $\eta$. A weak version of the next result can be founded in Lemma 2.1 in [5]. 
Theorem 2.5. Let $X$ be a topological space. The following facts are equivalents:

- The set of functions $\left\{K^{x}: x \in X\right\}$ is (locally) equicontinuous;

- $K$ is continuous;

- $\eta$ is continuous.

Proof. Let $z, w \in X$ and $K(z, w) \in \mathcal{O}$, where $\mathcal{O}$ is an open set in $\mathbb{C}$. If $\left\{K^{x}\right\}$ is (locally) equicontinuous and $\epsilon>0$, then there are open sets $z \in A, w \in B$ in $X$, for which

$$
|K(x, y)-K(x, w)|<\epsilon, \quad y \in B,
$$

and

$$
|K(x, w)-K(z, w)|=|K(w, x)-K(w, z)|<\epsilon, \quad x \in A
$$

Hence,

$$
|K(x, y)-K(z, w)| \leq|K(x, y)-K(x, w)|+|K(x, w)-K(z, w)|<2 \epsilon, \quad x \in A, y \in B .
$$

Pick $\epsilon>0$ such that the ball in $\mathbb{C}$ of radius $2 \epsilon$ centered in $K(z, w)$ is a subset of $\mathcal{O}$. It follows that

$$
K(A \times B) \subset \mathcal{O}
$$

and $K$ is continuous.

Now, we suppose that $K$ is continuous. Let $(x, y) \in X \times X$ and $\epsilon>0$. There are open sets $x \in U_{x}$ and $y \in U_{y}$ such that

$$
|K(x, y)-K(z, w)|<\epsilon, \quad(z, w) \in U_{x} \times U_{y}
$$

As so,

$$
|K(z, y)-K(z, w)| \leq|K(z, y)-K(x, y)|+|K(x, y)-K(z, w)|<2 \epsilon, \quad(z, w) \in U_{x} \times U_{y} .
$$

It follows that $\left\{K^{z}: z \in U_{x}\right\}$ is equicontinuous.

Also, if $A$ is an open set in $\mathcal{H}_{K}$ and $g=\eta(x) \in A$, for some $x \in X$. Let $\epsilon>0$ be such that the ball in $\mathcal{H}_{K}$ of radius $\sqrt{3 \epsilon}$ centered in $\eta(x)$ is a subset of $A$. There is an open set $\mathcal{O} \subset X$ for which $x \in \mathcal{O}$ and

$$
|K(x, x)-K(y, z)|<\epsilon, \quad y, z \in \mathcal{O} .
$$

It follows that

$$
|K(y, y)-K(y, x)| \leq|K(y, y)-K(x, x)|+|K(x, x)-K(y, x)|<2 \epsilon, \quad y \in \mathcal{O},
$$

and

$$
\|\eta(x)-\eta(y)\|_{K}^{2} \leq|K(x, x)-K(x, y)|+|K(y, y)-K(y, x)| \leq 3 \epsilon, \quad y \in \mathcal{O} .
$$

This clearly means that $\eta(\mathcal{O}) \subset A$ and $\eta$ is continuous. For the converse, we use the equality

$$
K(x, y)=\langle\eta(y), \eta(x)\rangle_{K}, \quad x, y \in X .
$$

If $f \in \mathcal{H}_{K}$ then $f(\cdot)=\langle f, \eta(\cdot)\rangle_{K}$ and a similar calculation leads to the continuity of $K$.

Corollary 2.6. If $K$ is continuous then $\mathcal{H}_{K} \subset C(X)$.

Corollary 2.7. If $X$ is a compact Haussdorf space, $\mathcal{F}$ is a bounded set in $\mathcal{H}_{K}$ and all $K^{x}$ are continuous. Then $\overline{\{\mathcal{F}\}}$ is compact in $C(X)$. Particularly, the inclusion map $i: \mathcal{H}_{K} \hookrightarrow C(X)$ is compact.

Proof. If $\mathcal{F}$ is bounded set in $\mathcal{H}_{K}$ then

$$
|f(x)| \leq M \sqrt{K(x, x)}, \quad x \in X, \quad f \in \mathcal{F}(X),
$$

and some $M>0$.

To guarantee the equicontinuity of $\mathcal{F}$ we just note that

$$
|f(x)-f(y)| \leq\left|\left\langle f, K^{x}-K^{y}\right\rangle_{K}\right| \leq\|f\|_{K}\left\|K^{x}-K^{y}\right\|_{K} .
$$

Since

$$
\left\|K^{x}-K^{y}\right\|_{K}=K(x, x)+K(y, y)-K(x, y)-K(y, x),
$$

the result follows from Arzelà-Ascoli theorem. 
If we also assume that $X$ is endowed with a convenient measure $\nu$ and the integral operator $\mathcal{K}$ : $L^{2}(X, \nu) \rightarrow L^{2}(X, \nu)$, given by

$$
\mathcal{K}(f)(x)=\int_{X} K(x, y) f(y) \mathrm{d} \nu(y), \quad f \in L^{2}(X, \nu), \quad x \in X,
$$

is well defined. If it is also bounded then we can find results dealing with the analysis of many questions related to $K$ and $\mathcal{K}$ in connection with the space $\mathcal{H}_{K}$. Note that if $\kappa$ belongs to $L^{1}(X, \nu)$ then inequality (2) ensures that $K^{x}$ belongs to $L^{2}(X, \nu)$ and $\mathcal{K}$ is a self-adjoint compact (Hilbert-Schmidt) operator.

An important issue that helps us to understand better a RKHS is to describe conditions under which it contains a copy of the range of $\mathcal{K}$. We will assume that $X$ is topological space endowed with a strict positive measure $\nu$, that is, a (complete) Borel measure for which two properties hold: every open nonempty subset of $X$ has positive measure and every $x \in X$ belongs to an open subset of $X$ with finite measure. The need for the assumptions above on $X$ and $\nu$ arises in technical arguments (see also $[7,8]$ for more details).

The first result needs the action of $\mathcal{K}$ on elements of $L_{c}^{2}(X, \nu)$, the set of all functions in $L^{2}(X, \nu)$ having compact support. As so, in this section we assume that $L_{c}^{2}(X, \nu)$ is dense in $L^{2}(X, \nu)$ or $\kappa$ belongs to $L^{1}(X, \nu)$. The proof we omit can be founded in [5].

Proposition 2.8. If every function in $\mathcal{H}_{K}$ is $\nu$-measurable then $\mathcal{K}\left(L^{2}(X, \nu)\right) \subset \mathcal{H}_{K}$ and

$$
\langle\mathcal{K}(f), g\rangle_{K}=\langle f, g\rangle_{2}, \quad g \in \mathcal{H}_{K}, \quad f \in L^{2}(X, \nu) .
$$

It is easy to see from the previous results that $K$ is $L^{2}(X, \nu)$-positive definite, that is,

$$
\langle\mathcal{K}(f), f\rangle_{2} \geq 0, \quad f \in L^{2}(X, \nu) .
$$

In that case, if the function $x \in X \rightarrow K^{x} \in L^{2}(X, \nu)$ is continuous and the formula

$$
\mathcal{K}(f)(x)=\left\langle f, K^{x}\right\rangle_{2}, \quad f \in L^{2}(X, \nu), \quad x \in X,
$$

shows that the range of $\mathcal{K}$ is a subset of $C(X)$. If $\mathcal{K}$ is a compact operator then a quite general version of Mercer's theorem along the lines of those proved in $[6,7]$ holds for $\mathcal{K}$. Precisely, $\mathcal{K}$ has spectral representation

$$
\mathcal{K}(f)=\sum_{n=1}^{\infty} \lambda_{n}\left\langle f, \phi_{n}\right\rangle_{2} \phi_{n}, \quad f \in L^{2}(X, \nu),
$$

with $\left\{\phi_{n}\right\}$ orthonormal in $L^{2}(X, \nu)$ and $\lambda_{n} \geq \lambda_{n+1} \rightarrow 0$. Also, $\left\{\lambda_{n} \phi_{n}\right\} \subset C(X)$ and

$$
K(x, y)=\sum_{n=1}^{\infty} \lambda_{n} \phi_{n}(x) \overline{\phi_{n}(y)}, \quad x, y \in X
$$

with absolute and uniform convergence on compact subsets of $X$.

These results enable us to prove the next important corollary (see [12] to another point of view). This result improves some results we have done in [8] and can be founded in our recent paper [4].

Corollary 2.9. Let us assume that $K$ has series representation like (4). Then it is strict $L^{2}$-positive definite, that is $\lambda_{n}>0, n=1,2, \ldots$, if, and only if, $\mathcal{H}_{K}$ is dense in $L^{2}(X, \nu)$.

Proof. Lemma 2.3 implies that $\left\{K^{x_{i}}\right\}$ is dense in $\mathcal{H}_{K}$ (for all dense set $\left\{x_{i}\right\}$ in $X$ ). Hence, if $f \in L^{2}(X, \nu)$ is orthogonal to $\left\{K^{x_{i}}\right\}$ then

$$
\mathcal{K}(f)\left(x_{i}\right)=\left\langle f, K^{x_{i}}\right\rangle_{2}=\left\langle\mathcal{K}(f), K^{x_{i}}\right\rangle_{K}=0,
$$

and follows that $\mathcal{K}(f)=0$. This means that $\mathcal{K}$ has no null eigenvalue if, and only if, $f=0$. By spectral theorem, this happens if, and only if, $\mathcal{H}_{K}$ is dense in $L^{2}(X, \nu)$. 
Remark 2.10. On the other side, the previous arguments also implies that $\left\{K^{x_{i}}\right\}$ is dense in $L^{2}(X, \nu)$ and $K$ is strict $L^{2}$-positive definite, if $\mathcal{K}$ has no null eigenvalue. As so, if

$$
0=g=\sum_{i=1}^{j} c_{i} K^{x_{i}}=\sum_{n=1}^{\infty}\left(\lambda_{n} \sum_{i=1}^{j} c_{i} \overline{\phi_{n}\left(x_{i}\right)}\right) \phi_{n}=\sum_{n=1}^{\infty}\left\langle g, \phi_{n}\right\rangle_{2} \phi_{n} .
$$

It follows that

$$
0=\sum_{i=1}^{j} c_{i} \overline{\phi_{n}\left(x_{i}\right)}
$$

But this doesn't looks sufficient to conclude that $K$ is strict positive definite. Results in [12] guarantee that the denseness of $\mathcal{H}_{K}$ in $C(X)$ is sufficient condition to the strict positive definiteness of $K$.

We finish this section with a way to construct reproducing kernels.

Let $\mathcal{F}(X)$ be a set of complex functions on $X$ and $\mathcal{H}$ a Hilbert space. Suppose there is a function $h: X \rightarrow \mathcal{H}$. Then we can define a linear transform $L$ given by

$$
L(f)(x)=\langle f, h(x)\rangle_{\mathcal{H}}, \quad x \in X, \quad f \in \mathcal{H} .
$$

This means that the evaluation functional $\delta_{x}$ is bounded on the range of $L$.

Lemma 2.11. The completion of the range of $L$ is a RKHS with kernel

$$
K(x, y)=\langle h(y), h(x)\rangle_{\mathcal{H}}, \quad x, y \in X .
$$

Also, $L: \mathcal{H} \rightarrow \overline{L(\mathcal{H})}$ has norm 1 .

Proof. We first note that if $f \in\{h(x): x \in X\}^{\perp}$ then $L(f)=0$. Also,

$$
f=\sum_{i=1}^{n} c_{i} h\left(x_{i}\right) \Longrightarrow L(f)(x)=\sum_{i=1}^{n} c_{i}\left\langle h\left(x_{i}\right), h(x)\right\rangle_{\mathcal{H}}=\sum_{i=1}^{n} c_{i} K\left(x, x_{i}\right)=u(x) .
$$

We can then see that

$$
K(x, y)=\langle h(y), h(x)\rangle_{H}, \quad x, y \in X,
$$

is the reproducing kernel of $L(\mathcal{H})$. Particularly, this means that

$$
\|f\|_{\mathcal{H}}=\sum_{i, j=1}^{n} c_{i} \overline{c_{j}}\left\langle h\left(x_{i}\right), h\left(x_{j}\right)\right\rangle_{\mathcal{H}}=\sum_{i, j=1}^{n} c_{i} \overline{c_{j}} K\left(x_{i}, x_{j}\right)=\|u\|_{K}=\|L(f)\|_{K} .
$$

It follows that $L: \mathcal{H} \rightarrow \overline{L(\mathcal{H})}$ has norm 1 .

Corollary 2.12. $\operatorname{Ker}(L)=\{h(x): x \in X\}^{\perp}, i$. e. $\overline{\operatorname{span}\{h(x): x \in X\}}=\operatorname{Ker}(L)^{\perp}$.

Proof. We saw that if $f \in\{h(x): x \in X\}^{\perp}$ then $L(f)=0$, this means that $\operatorname{Ker}(L) \supset\{h(x): x \in X\}^{\perp}$. But, if $L(f)=0$ then it is clear that $f \perp h(x)$, for all $x \in X$. It follows that $\overline{\operatorname{span}\{h(x): x \in X\}}=$ $\operatorname{Ker}(L)^{\perp}$.

Corollary 2.13. If $u \in \mathcal{H}_{K}=\overline{L(\mathcal{H})}$, then the unique $f \in \mathcal{H}$, with $\|f\|_{\mathcal{H}}=\|u\|_{K}$, is $L^{*}(u)$.

Proof. Note that, if $u=L(f)$ then

$$
u(x)=\left\langle u, K^{x}\right\rangle_{K}=\left\langle L(f), K^{x}\right\rangle_{K}=\left\langle f, L^{*}\left(K^{x}\right)\right\rangle_{\mathcal{H}}=\langle f, h(x)\rangle_{\mathcal{H}} .
$$

It follows that

$$
L^{*}\left(K^{x}\right)=h(x), \quad x \in X
$$

Equation 5 implies that

$$
L^{*} L(f)=f
$$

and $L^{*}$ acts like the inverse of $L$. But if $g \in\{h(x): x \in X\}^{\perp}$ then $L(g+f)=L(f)=u$ and the result follows. 
Corollary 2.14. If $\mathcal{H}$ is a $R K H S$ with reproducing kernel $R: X \times X \rightarrow \mathbb{C}$ then

$$
L^{*}(u)(x)=f(x)=\left\langle L^{*} u, R^{x}\right\rangle_{\mathcal{H}}=\left\langle L^{*} u, R^{x}\right\rangle_{R}=\left\langle u, L R^{x}\right\rangle_{K}, \quad u \in \mathcal{H}_{K} .
$$

Remark 2.15 (Laplace transform). If $f \in L^{2}(0,+\infty)$ then its Laplace transform is given by

$$
\mathfrak{L}[f](s)=\int_{0}^{+\infty} e^{-s t} f(t) \mathrm{d} t=\langle f, h(s)\rangle_{L^{2}}, \quad 0<s<\infty,
$$

where $h(x)(y)=e^{-x y}, x, y \in(0,+\infty)$. The range of $\mathfrak{L}$ is a RKHS with reproducing kernel

$$
K(x, y)=\int_{0}^{+\infty} e^{-x t} e^{-y t} \mathrm{~d} t=\frac{1}{x+y}, \quad x, y \in(0,+\infty)
$$

Please take a look at [9] to application of this arguments on initial value problems.

\section{On the Solution of Equations on RKHS}

At the beginning of this work we talk about reproducing kernel Hilbert spaces in a natural way. These spaces are our point of interest in writing this paper, because of his wide class of application (see $[5,6,10,12])$.

Until the end of his section we discuss some facts about solutions of linear equations of the form

$$
L(f)(x)=u(x), \quad x \in X, \quad f \in \mathcal{H}, \quad u \in \mathcal{H}_{K},
$$

where $L$ is a bounded linear operator. It follows from reproducing property that

$$
L(f)(x)=\left\langle L(f), K^{x}\right\rangle_{K}=\left\langle f, L^{*} K^{x}\right\rangle_{\mathcal{H}}=u(x), \quad x \in X, \quad f \in \mathcal{H}, \quad u \in \mathcal{H}_{K}
$$

where $L^{*}$ is the adjoint of $L$.

In a first look we can think that this procedure is just useful to work with linear equations. But this is not the case. You can see the paper [13] to a special case of equation

$$
L(f)(x)=u(x)=G(x, f(x))
$$

\section{1 $\mathcal{H}=\mathcal{H}_{R}$ is a Reproducing Kernel}

To start this discussion we use Lemma 2.3, and assume that $\mathcal{H}=\mathcal{H}_{R}$ is also a reproducing kernel.

Lemma 3.1. Let $L: \mathcal{H}_{R} \rightarrow \mathcal{H}_{K}$ be a bounded linear operator, with $\mathcal{H}_{R} \subset C(X)$. If $\left\{x_{i}\right\}_{i \in A}$ is dense in $X$ then $\operatorname{span}\left\{L\left(R^{x_{i}}\right)\right\}_{i \in A}$ is dense in $\mathcal{H}_{K}$ if, and only if, $L$ has a dense range. Particularly, if $L$ is one to one and $R$ is strict positive definite then $\left\{L\left(R^{x_{i}}\right)\right\}_{i \in A}$ is linearly independent.

Proof. It follows from Lemma 2.3 that $\operatorname{span}\left\{R^{x_{i}}\right\}_{i \in A}$ is dense in $\mathcal{H}_{R}$. If $L$ has a dense range in this space, then all function $g$ in $\mathcal{H}_{K}$ has a function $h=L f$, as close as you need of it, to some $f \in \mathcal{H}_{R}$. It follows that there exists $q$ in $\operatorname{span}\left\{R^{x_{i}}\right\}_{i \in A}$, as close as you want of $f$. As so $L q$ is in $\operatorname{span}\left\{L\left(R^{x_{i}}\right)\right\}_{i \in A}$ and as close as you need of $h$. The proof follows.

Corollary 3.2. Let $L: \mathcal{H}_{R} \rightarrow \mathcal{H}_{K}$ be a bounded injective linear operator, with $\mathcal{H}_{R} \subset C(X)$. If $\left\{x_{i}\right\}_{i \in A}$ is dense in $X$ and $R$ is strict positive definite, then $\left\{L\left(R^{x_{i}}\right)\right\}_{i \in A}$ is linearly independent in $\mathcal{H}_{K}$. If $A \subset \mathbb{N}$ then you can orthonormalize this set to construct $\left\{\Phi_{i}\right\}$ as a basis to the range of L, by using Gram-Shmidt process.

We may use the next result to understand how to handle with $L^{*}$. 
Corollary 3.3. Let $L: \mathcal{H}_{R} \rightarrow \mathcal{H}_{K}$ be a bounded one to one linear operator, with $\mathcal{H}_{K} \subset C(X)$. If $\left\{x_{i}\right\}_{i \in A}$ is dense in $X$ then $\operatorname{span}\left\{L^{*}\left(K^{x_{i}}\right)\right\}_{i \in A}$ is dense in $\mathcal{H}_{R}$. Particularly, if $K$ is strict positive definite then $\left\{L^{*}\left(K^{x_{i}}\right)\right\}_{i \in A}$ is linearly independent. If $A \subset \mathbb{N}$ then you can find an orthonormal basis $\left\{\Psi_{i}\right\}$ to $\mathcal{H}_{R}$, where

$$
\Psi_{i}=\sum_{j=1}^{i} \beta_{j i} L^{*} K^{x_{j}}
$$

by using Gram-Shmidt process. Also,

$$
L f\left(x_{i}\right)=\left\langle L f, K^{x_{i}}\right\rangle_{K}=\left\langle f, L^{*} K^{x_{i}}\right\rangle_{R}, \quad f \in \mathcal{H}_{R},
$$

and

$$
L^{*} K^{x_{j}}(x)=\left\langle L^{*} K^{x_{i}}, R^{x}\right\rangle_{R}=\left\langle K^{x_{i}}, L R^{x}\right\rangle_{K}=\overline{L R^{x}\left(x_{i}\right)} .
$$

Now we can express the solution of (1), in this context, by using Parseval's identity.

Theorem 3.4. If $u(x)=L f(x)=G(x, f(x))$, with $f \in \mathcal{H}_{R}$, has only one solution, for all $u \in \mathcal{H}_{K}$. Under conditions of Corollary 3.3 it holds,

$$
f(x)=\sum_{i=1}^{\infty} \alpha_{i} \Psi_{i}(x)
$$

with uniform convergence, where

$$
\alpha_{i}=\left\langle f, \Psi_{i}\right\rangle_{R}=\left\langle f, \sum_{j=1}^{i} \beta_{j i} L^{*} K^{x_{j}}\right\rangle_{R}=\left\langle L f, \sum_{j=1}^{i} \beta_{j i} K^{x_{j}}\right\rangle_{K}=\sum_{j=1}^{i} \overline{\beta_{j i}} L f\left(x_{j}\right)=\sum_{j=1}^{i} \overline{\beta_{j i}} G\left(x_{j}, f\left(x_{j}\right)\right) .
$$

Problems like (2) motivates the next result.

Corollary 3.5. If $L f(x)=g(x)$ is a given function then

$$
f(x)=\sum_{i=1}^{\infty}\left(\sum_{j=1}^{i} \beta_{j i} g\left(x_{j}\right)\right) \Psi_{i}(x)
$$

with uniform convergence.

We can rewrite results in this section to work with systems of equations (see [1], for instance).

\section{Application to Volterra Integral Equations Theory}

In this section we analyze some previous results to enable one to find the solution of Volterra integral equations like

$$
L f(x)=A(x) f(x)+\int_{0}^{x} H(x, t) G(f(t)) \mathrm{d} t=u(x),
$$

with given continuous functions

$$
A(x)=\left[a_{i j}(x)\right]_{n \times n}, H(x, t)=\left[h_{i j}(x, t)\right]_{n \times n}, f(x)=\left[f_{i}(x)\right]_{n \times 1}, u(x)=\left[u_{i}(x)\right]_{n \times 1}, \quad t, x \in[0,1] .
$$

We suppose that this equation has a unique solution $f:[0,1] \rightarrow \mathbb{R}^{n}$ (this section has glimpses from $[1,11,13]$ ). We assume (by simplicity) $G(f(t))=C(t) f(t)$, with $C(t)=\left[c_{i j}(t)\right]_{n \times n}$ (we may look it as an approximation to the nonlinear case $G(u)=c_{1}+c_{2} u+r(u)$, with $|r(u)| /|u| \simeq 0$ if $\left.u \simeq 0\right)$. We can then rewrite (2) as

$$
A(x) f(x)+\int_{0}^{x} H(x, t) f(t) \mathrm{d} t=u(x)
$$


Note that, if $A(x)$ has inverse on $[0,1]$, then this equation is like

$$
f(x)+\int_{0}^{x} H(x, t) f(t) \mathrm{d} t=u(x) .
$$

It is clearly that the operator $T_{u}: C[0,1] \rightarrow C[0,1]$, given by

$$
T_{u}(f)(x)=u(x)-\int_{0}^{x} H(x, t) f(t) \mathrm{d} t,
$$

is well defined and we can apply Banach fixed point theorem to $T_{u}^{j}$, for some big $j \in \mathbb{N}$, to see that there exists a unique continuous function $f$, such that

$$
T_{u}(f)(x)=f(x), \quad x \in[0,1] .
$$

\subsection{Choosing a Reproducing Kernel}

Suppose now that $H$ is continuous and $\partial H / \partial x$ is in $L^{2}[0,1]$. Denote by $W[0,1]$ the space of absolutely continuous functions $f$, with $f^{\prime} \in L^{2}[0,1]$ and inner product given by

$$
\langle f, g\rangle_{W}=f(0) g(0)+\int_{0}^{1} f^{\prime}(x) g^{\prime}(x) \mathrm{d} x .
$$

We can show that the functions $u$ in $W[0,1]$ have the form

$$
u(x)=u(0)+\int_{0}^{x} u^{\prime}(s) \mathrm{d} s, \quad x \in[0,1] .
$$

Denote also by $W[0,1]$ the space of functions $f(x)=\left(f_{1}(x), f_{2}(x), \ldots, f_{n}(x)\right), x \in[0,1]$, with $f_{i} \in W[0,1]$, for all $i=1,2, \ldots, n$, and inner product with similar form, where $f(x) g(x)$ denote the usual inner product of $f(x)$ and $g(x)$ in $\mathbb{R}^{n}$.

Remark 4.1. Since all polynomial function is in $W[0,1]$, it follows that this set is dense in $C[0,1]$. This is a very important property of a space in approximation theory $([12])$.

It is clear that

$$
\frac{d T_{u}(f)(x)}{\mathrm{d} x}=u^{\prime}(x)-H(x, x) f(x)-\int_{0}^{x} \frac{\partial H(x, t)}{\partial x} f(t) \mathrm{d} t .
$$

This means that if $u \in W[0,1]$ then $T_{u}(f) \in W[0,1]$ and we can write $T_{u}: C[0,1] \rightarrow W[0,1]$. Hence, $T_{u}(f)=f$ has a unique solution in $W[0,1]$. This means that $T_{u}: W[0,1] \rightarrow W[0,1]$, has a unique fixed point, if $u \in W[0,1]$.

Now we can then define the bijective linear operator $L: W[0,1] \rightarrow W[0,1]$, given by

$$
L(f)(x)=f(x)+\int_{0}^{x} H(x, t) f(t) \mathrm{d} t .
$$

The problem of finding the fixed point of $T_{u}$ is the same as to solve the functional linear equation $L(f)=u$, for a given $u \in W[0,1]$. Particularly

$$
T_{u}(f)=f \Longleftrightarrow L(f)=u \text {. }
$$

The next result is to guarantee that each coordinate of functions in $W[0,1]$ is in a reproducing kernel Hilbert space with strict positive definite kernel

$$
K(x, y)=1+\min (x, y)=\left\{\begin{array}{ll}
1+y, & x \geq y \\
1+x, & x<y
\end{array} .\right.
$$

This enables one to use the arguments in Section 2 to approximate solution of Volterra integral equations, by using a numerical procedure to calculate the $\alpha_{i}$ in expressions of Theorem 3.4 and truncating the series. 
Lemma 4.2. If $f \in W[0,1]$ then $|f(x)| \leq(1+\sqrt{x})\|f\|_{W}$.

Proof. We put $f_{i}(x)=f_{i}(0)+\int_{0}^{x} f_{i}^{\prime}(s) \mathrm{d} s$ for each coordinate of $f \in W[0,1]$. Hence $f(x)=f(0)+\int_{0}^{x} f^{\prime}(s) \mathrm{d} s$. It follows that

$$
|f(x)| \leq|f(0)|+\int_{0}^{x}\left|f^{\prime}(s)\right| \mathrm{d} s .
$$

But

$$
|f(0)| \leq \sqrt{|f(0)|^{2}+\int_{0}^{1}\left|f^{\prime}(s)\right|^{2} \mathrm{~d} s}=\|f\|_{W}
$$

and

$$
\int_{0}^{x}\left|f^{\prime}(s)\right| \mathrm{d} s \leq \sqrt{x \int_{0}^{1}\left|f^{\prime}(s)\right|^{2} \mathrm{~d} s} \leq \sqrt{x} \sqrt{|f(0)|^{2}+\int_{0}^{1}\left|f^{\prime}(s)\right|^{2} \mathrm{~d} s}=\sqrt{x}\|f\|_{W} .
$$

Lemma 4.3. The operator $L: W[0,1] \rightarrow C[0,1]$ is bounded.

Proof. Lema 4.2 implies that, if $f \in W[0,1]$ then $|f(x)| \leq 2\|u\|_{W}$ and hence

$$
|L(f)(x)| \leq 2\left(1+\int_{0}^{x}|H(x, t)| \mathrm{d} t\right)\|f\|_{W}
$$

Lemma 4.4. $W[0,1]$ is a Hilbert space.

Proof. Let $\left\{f_{j}\right\}$ be a Cauchy sequence in $W[0,1]$. As so, given $\varepsilon>0$, there exists $J \in \mathbb{N}$ such that if $j, l>J$, then

$$
\left\|f_{j}-f_{l}\right\|_{W}^{2}=\left|f_{j}(0)-f_{l}(0)\right|^{2}+\int_{0}^{1}\left|f_{j}^{\prime}(x)-f_{l}^{\prime}(x)\right|^{2} \mathrm{~d} x<\varepsilon .
$$

Previous lemma implies that

$$
\left|f_{j}(x)-f_{l}(x)\right| \leq 2\left\|f_{j}-f_{l}\right\|_{W}<\varepsilon .
$$

Since each coordinate of $f_{j}$ and $f_{j}^{\prime}$ is in $C[0,1]$ and $L^{2}[0,1]$ respectively, which are Cauchy sequences, there exist $f$ and $g$, with coordinates in $C[0,1]$ and $L^{2}[0,1]$ respectively, such that $f_{j} \rightarrow f$ uniformly and

$$
\int_{0}^{1}\left|f_{j}^{\prime}(x)-g(x)\right|^{2} \mathrm{~d} x \rightarrow 0 .
$$

If

$$
h(x)=f(0)+\int_{0}^{x} g(s) \mathrm{d} s
$$

which is uniformly continuous, then

$$
\left\|f_{j}-h\right\|_{W}^{2}=\left|f_{n}(0)-f(0)\right|^{2}+\int_{0}^{1}\left|f_{j}^{\prime}(x)-g(x)\right|^{2} \mathrm{~d} x \rightarrow 0
$$

and $f=h$ a.e..

It follows that $W[0,1]$ is a Hilbert space.

Theorem 4.5. If $W[0,1]$ is a set of scalar functions then it is a reproducing kernel Hilbert space with kernel

$$
K(x, y)=1+\min (x, y)=\left\{\begin{array}{ll}
1+y, & x \geq y \\
1+x, & x<y
\end{array} .\right.
$$


Proof. It follows from Lemma 4.2 that, for all $x \in[0,1]$, the linear functional $\delta_{x}: W[0,1] \rightarrow \mathbb{R}$, given by $\delta_{x}(f)=f(x)$ is bounded. Since $W[0,1]$ is a Hilbert space, it follows that it is a RKHS.

Since $u_{y}=K^{y}$ is continuous with derivative

$$
u_{y}^{\prime}(x)=\left\{\begin{array}{ll}
0, & x \geq y \\
1, & x<y
\end{array},\right.
$$

in $L^{2}[0,1]$. This means that

$$
u_{y}(x)=u_{y}(0)+\int_{0}^{x} u_{y}^{\prime}(s) \mathrm{d} s
$$

is absolutely continuous in $[0,1]$.

On the other side,

$$
\begin{aligned}
\left\langle f, K^{x}\right\rangle_{W} & =f(0) K(0, x)+\int_{0}^{1} f^{\prime}(s) u_{x}^{\prime}(s) \mathrm{d} s \\
& =f(0)(1+0)+\int_{0}^{x} f^{\prime}(s) u_{x}^{\prime}(s) \mathrm{d} s+\int_{x}^{1} f^{\prime}(s) u_{x}^{\prime}(s) \mathrm{d} s \\
& =f(0)+\int_{0}^{x} f^{\prime}(s) \mathrm{d} s \\
& =f(x)
\end{aligned}
$$

The proof follows.

Corollary 4.6. If $f=\left(f_{1}, f_{2}, \ldots, f_{n}\right) \in W[0,1]$ then

$$
f_{i}(x)=\left\langle f_{i}, K^{x}\right\rangle_{W}, \quad x \in[0,1] .
$$

Corollary 4.7. If $f \in W[0,1]$ and

$$
\mathbf{K}=\left[\begin{array}{cccc}
K & 0 & \ldots & 0 \\
0 & K & \ldots & 0 \\
\vdots & \vdots & \ddots & \vdots \\
0 & 0 & \ldots & K
\end{array}\right]_{n \times n}
$$

then

$$
f(x) v=\left\langle f, \mathbf{K}^{\mathbf{x}} v\right\rangle_{W}, \quad f \in W[0,1], \quad v \in \mathbb{R}^{n} .
$$

Since $K$ in the previous result is a reproducing kernel, it follows that it is positive definite. To prove the next result about the strict positiveness of $K$, we need the following lemma.

Lemma 4.8. If $A=\left[a_{i, j}\right]_{l \times l}$, with

$$
a_{i j}=\min \left(b_{i}, b_{j}\right)=\left\{\begin{array}{l}
b_{i}, i<j \\
b_{j}, i \geq j
\end{array}, \quad 0<b_{1}<b_{2}<\cdots<b_{l},\right.
$$

then $A$ is strict positive definite.

Proof. If $A=\left[b_{1}\right]$ then it is clearly (strict) positive definite. Suppose by a inductive argument that it holds for some $j \geq 1$ and let $l=j+1$. It follows from Chiï $i \frac{1}{2}$ 's rule that

$$
\operatorname{det} A=b_{1}^{l} \operatorname{det}\left[\begin{array}{cccc}
b_{2} / b_{1}-1 & b_{2} / b_{1}-1 \ldots & b_{2} / b_{1}-1 \\
b_{2} / b_{1}-1 & b_{3} / b_{1}-1 \ldots & b_{3} / b_{1}-1 \\
\vdots & \ddots & \vdots & \vdots \\
b_{2} / b_{1}-1 & b_{3} / b_{1}-1 \ldots & b_{l} / b_{1}-1
\end{array}\right]>0 .
$$

The proof follows from the fact that a matrix is (strict) positive definite if, and only if, all principal submatrix is (strict) positive definite, which means that its determinant positive. 
We may use Corollary 2.9 to guarantee that $K$ is strict $L^{2}$-positive definite (see also Corollary 2.2 in [8], Corollary 7 in [12] or [4]). But we prove this here directly.

Theorem 4.9. $K$ is a strict $L^{2}$-positive and positive definite kernel on $[0,1]^{2}$.

Proof. Let $f \in C[0,1]$ be a non null function. By simplicity we assume that $f$ is a real function. Denote by

$$
F(x)=\int_{0}^{x} f(s) \mathrm{d} s, \quad G(x)=\int_{0}^{x} F(s) \mathrm{d} s .
$$

As so,

$$
\int_{0}^{x} y f(y) \mathrm{d} y=x F(x)-G(x), \quad \int_{0}^{x} f(s) G(s) \mathrm{d} s=F(x) G(x)-\int_{0}^{x}[F(x)]^{2} \mathrm{~d} x,
$$

and

$$
|G(1)|^{2}=\left|\int_{0}^{1} F(x) \mathrm{d} x\right|^{2} \leq \int_{0}^{1}|F(x)|^{2} \mathrm{~d} x
$$

It follows that

$$
\begin{aligned}
\int_{0}^{1} \int_{0}^{1} K(x, y) f(x) f(y) \mathrm{d} x \mathrm{~d} y & =\int_{0}^{1} \int_{0}^{1} f(x) f(y) \mathrm{d} x \mathrm{~d} y+\int_{0}^{1} \int_{0}^{x} y f(x) f(y) \mathrm{d} y \mathrm{~d} x \\
& +\int_{0}^{1} \int_{x}^{1} x f(x) f(y) \mathrm{d} y \mathrm{~d} x \\
& =F(1)^{2}+\int_{0}^{1} f(x) \int_{0}^{x} y f(y) \mathrm{d} y \mathrm{~d} x+\int_{0}^{1} x f(x) \int_{x}^{1} f(y) \mathrm{d} y \mathrm{~d} x \\
& =F(1)^{2}+\int_{0}^{1} f(x)[x F(x)-G(x)] \mathrm{d} x+\int_{0}^{1} x f(x)[F(1)-F(x)] \mathrm{d} x \\
& =F(1)^{2}-\int_{0}^{1} f(x) G(x) \mathrm{d} x+F(1) \int_{0}^{1} x f(x) \mathrm{d} x \\
& =F(1)^{2}-\left[F(1) G(1)-\int_{0}^{1}[F(x)]^{2} \mathrm{~d} x\right]+F(1)[F(1)-G(1)] \\
& =2 F(1)^{2}-2 F(1) G(1)+\int_{0}^{1}[F(x)]^{2} \mathrm{~d} x \\
& =[F(1)-G(1)]^{2}+|F(1)|^{2}+\int_{0}^{1}[F(x)]^{2} \mathrm{~d} x-|G(1)|^{2} \\
& \geq[F(1)-G(1)]^{2}+|F(1)|^{2}
\end{aligned}
$$

If $2 F(1)^{2}-2 F(1) G(1)+\int_{0}^{1}[F(x)]^{2} \mathrm{~d} x=0$, then (particularly) $F(1)=G(1)=0$ and $\int_{0}^{1}[F(x)]^{2} \mathrm{~d} x=0$. This means that $F(x)=0$ and this implies $f(x)=0$ (one thing that can't happens). The proof of (strict) $L^{2}$-positive definiteness follows.

Now, let $j \in \mathbb{N}$ and $x_{1}<x_{2}<\cdots<x_{j} \in[0,1]$. It follows that

$$
\left[K\left(x_{i}, x_{j}\right)\right]=\left[\begin{array}{cccc}
1+x_{1} & 1+x_{1} & \ldots & 1+x_{1} \\
1+x_{1} & 1+x_{2} & \ldots & 1+x_{2} \\
\vdots & \vdots & \ddots & \vdots \\
1+x_{1} & 1+x_{2} & \ldots & 1+x_{j}
\end{array}\right]
$$

The strict positive definiteness of $K$ follows from the previous Lemma.

To finish this section and the paper we write two examples of RKHS. The same argument of Remark 4.1 may be used to see that the first one is dense in $C[0,1]$. We can then apply Corollary 2.9 to guarantee that it is strict $L^{2}$-positive definite or use results in [12] to see that this kernel is strict positive definite. 
Remark 4.10. We denote by $W_{j}[0,1]$ the space of functions $f:[0,1] \rightarrow \mathbb{C}$ with $f^{\prime}, f^{\prime \prime}, \ldots, f^{(j-1)}$ absolutely continuous and $f^{(j)} \in L^{2}[0,1]$. If this space is endowed with the inner product

$$
(f, g)_{W_{j}}=f(0) g(0)+f^{\prime}(0) g^{\prime}(0)+\cdots+f^{(j-1)}(0) g^{(j-1)}(0) \int_{0}^{1} f^{(j)}(x) g^{(j)}(x) \mathrm{d} x,
$$

then it is a RKHS.

If $j=2$, the kernel is

$$
R(x, y)= \begin{cases}1+x y+\frac{x y^{2}}{2}-\frac{y^{3}}{6}, & y \leq x \\ 1+x y+\frac{x^{2} y}{2}-\frac{x^{3}}{6}, & y>x\end{cases}
$$

Next we give an example of RKHS with a non strictly $L^{2}$-positive definite kernel.

Remark 4.11. The subspace $\mathcal{H} \subset W[0,1]$, with $f(0)=f(1)$, is a RKHS with Mercer kernel

$$
K_{2}(x, y)=\min (x, y)-x y=\left\{\begin{array}{ll}
y(1-x), & y \leq x \\
x(1-y), & y>x
\end{array}, \quad x, y \in[0,1] .\right.
$$

Direct calculations show that the associated integral operator has

$$
\lambda_{n}=\frac{1}{n^{2} \pi^{2}}, \quad \phi_{n}(x)=\operatorname{sen}(n \pi x), \quad n=1,2, \ldots,
$$

as eigenvalues and eigenfunctions respectively. Since $\left\{\phi_{n}\right\}$ is orthonormal but not a basis to $L^{2}[0,1]$, we can see that $\mathcal{H}$ is not dense there and that 0 is an eigenvalue. This means, by Corollary 2.9 , that $K$ is not strict positive definite.

Acknowledgments. The author was partially supported by Fundação de Amparo à Pesquisa do Estado de Minas Gerais - FAPEMIG and CNPq Brasil.

\section{References}

1. Wei Jiang, Zhong Chen, Solving a system of linear Volterra integral equations using the new reproducing kernel method, Applied Mathematics and Computation 219 (2013).

2. F. Cucker, S. Smale, On the mathematical foundations of learning. Bull. Amer. Math. Soc. (N.S.) 39 (2002), no. $1,1-49$.

3. R. Cavoretto, G. E. Fasshauer, M. Mccourt, An introduction to the Hilbert-Schmidt SVD using iterated Brownian bridge kernels. Numer. Algor. (2014).

4. J. C. Ferreira, On reproducing kernel and density problems, Positivity (2017) 21: 143. doi:10.1007/s11117-0160414-4.

5. J. C. Ferreira, V. A. Menegatto, Positive definiteness, reproducing kernel Hilbert spaces and beyond, AFA, 64-88, 2013.

6. J. C. Ferreira, V. A. Menegatto, Reproducing properties of differentiable Mercer-like kernels, Math. Nach., 285, no. 8-9, 959-973, 2012, DOI 10.1002/mana.201100072.

7. J. C. Ferreira, V. A. Menegatto, Eigenvalue decay rates for positive integral operators, Annali di Matematica (2013), 1025-1041, DOI 10.1007/s10231-012-0256-z.

8. J. C. Ferreira, V. A. Menegatto, Eigenvalues of integral operators defined by smooth positive definite kernels, Integral Equations and Operator Theory, 64 (2009), no. 1, 61-81.

9. L. P. Castro, M. M. Rodrigues, S. Saitoh, A Fundamental Theorem on Initial Value Problems by Using the Theory of Reproducing Kernels, Complex Analysis and Operator Theory, 2014.

10. H. Sun; Q. Wu, Application of integral operator for regularized least-square regression. Math. Comput. Modelling, 49 (2009), 276-285.

11. Li-Hong Yang, Hong Ying Li, Jing-Ran Wang, Solving a system o linear Volterra integral equation using the modified reproducing kernel method, Abstract and Applied Analysis, Article ID 196308, 2013.

12. D.-X. Zhou, Density Problem and Approximation Error in Learning Theory. Abstract and Applied Analysis Volume 2013, Article ID 715683.

13. O. A. Arqub, M. Al-Smadi, S. Momani, Application of Reproducing Kernel Method for Solving Nonlinear Fredholm-Volterra Integrodifferential Equations, Abstract and Applied Analysis, Volume 2012, Article ID 839836. 